\title{
Optimal Debt Ratio and Dividend Payment Strategies with Reinsurance
}

\author{
Zhuo Jin, ${ }^{*} \quad$ Hailiang Yang, ${ }^{\dagger} \quad$ G. Yin ${ }^{\ddagger}$
}

June 2, 2015

\begin{abstract}
This paper derives the optimal debt ratio and dividend payment strategies for an insurance company. Taking into account the impact of reinsurance policies and claims from the credit derivatives, the surplus process is stochastic that is jointly determined by the reinsurance strategies, debt levels, and unanticipated shocks. The objective is to maximize the total expected discounted utility of dividend payment until financial ruin. Using dynamic programming principle, the value function is the solution of a second-order nonlinear Hamilton-Jacobi-Bellman equation. The subsolution-supersolution method is used to verify the existence of classical solutions of the Hamilton-Jacobi-Bellman equation. The explicit solution of the value function is derived and the corresponding optimal debt ratio and dividend payment strategies are obtained in some special cases. An example is provided to illustrate the methodologies and some interesting economic insights.
\end{abstract}

Key Words. Stochastic control, reinsurance policies, optimal debt ratio, dividend strategies, financial crisis.

${ }^{*}$ Centre for Actuarial Studies, Department of Economics, The University of Melbourne, VIC 3010, Australia, zjin@unimelb.edu.au.

${ }^{\dagger}$ Department of Statistics and Actuarial Science, The University of Hong Kong, Hong Kong, hlyang@hku.hk.

${ }^{\ddagger}$ Department of Mathematics, Wayne State University, Detroit, Michigan 48202, gyin@math.wayne.edu.

(C) 2015. This manuscript version is made available under the Elsevier user license http://www.elsevier.com/open-access/userlicense/1.0/ 


\section{Introduction}

Since the collapse of US housing market in 2007, which led the initial sub-prime mortgage crisis into a global financial crisis in 2008, financial/insurance institutions and regulators have drawn increasing attention to evaluate and monitor risk so as to avoid insolvency. In this paper we analyze the failure of American International Group (AIG) in the global financial crisis. Our work focuses on AIG that sold Credit Default Swaps (CDSs), a form of insurance, against the financial risks that were based upon debt from the real estate market. At its peak, AIG was one of the largest and most successful companies in the world boasting a Triple-A credit rating, over $\$ 1$ trillion in assets, and 76 million customers in more than 130 countries. AIG occupied an important role in the financial system. However, due to poor risk management structure, combined with a lack of regulatory oversight, AIG accumulated substantial amounts of risk and unsustainable insurance liabilities by issuing large amount of CDSs, which led to the crash of the insurance giant.

During 2001-2006, the low interest rates and rises in housing prices induced a substantial demand for mortgages. However, as financial institutions were chasing for higher returns, the leverage tools were overused and quality of mortgages declined. Mortgage originators such as Countrywide sell packages of mortgages to the major banks. The latter securities firms in turn structure the packages and tranche them into senior, mezzanine and equity tranches. The securities firms then sell the collateralized debt obligations (CDOs) to international investors, hedge funds and investment banks such as Merrill Lynch, Citi-group and Goldman-Sachs. If the mortgagors are unable to service their debts, the income from the mortgages declines. The cash flows all along the line will suffer. Securities firms and hedge funds may buy CDSs from companies such as AIG as insurance against depreciation in the values of the CDOs. When the market is highly leveraged, the financial system becomes vulnerable since the small change in asset values will significantly influence the net wealth. After the housing price peaked in early 2006, the bursting of housing bubble resulted in the credit and liquidity crisis and the recession thereafter. AIG Financial Product (AIGFP), a subsidiary of AIG, entered the credit derivatives market in 1998 when it underwrote its first CDS with JP Morgan. Over time AIGFP became a central player in the fast-growing CDS market. AIGFP's corporate arbitrage CDS portfolio was comprised of CDS contracts written on CDOs. The collateral pools backing the multi-sector CDOs included prime, Alt-A, and subprime residential mortgage-backed securities (RMBS), commercial mortgage-backed securities (CMBS), other asset-backed securities (ABS). In many cases non-agency CDOs are required to carry insurance in order to obtain a high credit rating. The CDSs are privately negotiated contracts that perform in a similar manner to insurance contacts, but their payoff function is similar to a put option. The CDS requires that the insurer put up more collateral if the market value of the securities insured falls below the predetermined level. Claims are the required payments to the insured holders of CDSs, due to either defaults of the obligors or for collateral calls when the prices of the insured securities decline. However, AIG didn't set sufficient surplus aside to cope with the collateral claims, which led to the catastrophe of AIG and the biggest corporate bailout in US history.

The CDSs insured by AIG were ultimately related to the systemic risk from the inability of the mortgagors to service their debts. AIG made a series of serious mistakes in the risk management. Risk was underestimated because AIG ignored the negative correlation between the investment income and the claims. The estimate of the drift of the capital gain was based on the unsustainable growth of the housing price index during the era of booming house market. Taking into account the financial leverage effect, a collapse would occur when the unsustainable capital gain sunk below the interest rate. The CDS claims surged when the value of the insured securities declined. This triggered additional collateral requirements, and the stability and credit rating of AIG was 
undermined. From AIG's case, we can see that it is of great importance to discuss the optimal debt level and amount of insurance liabilities an insurance company could offer. Meng et al. (2013) considered an optimal dividend problem with nonlinear insurance risk processes attributed to internal competition factors, and incorporated other important features such as the presence of debts and transaction costs. On the other hand, in insurance companies, insurers tend to accumulate relatively large amounts of cash, cash equivalents, and pursue capital gains in order to pay future claims and avoid financial ruin because of the nature of their insurance product. The payment of dividends to shareholders may reduce an insurer's ability to survive adverse investment and underwriting experience. The study of optimizing the stream of dividend payments and management of surplus is a high priority task. Initiated in the work of De Finetti (1957), there have been increasing efforts on using advanced methods of stochastic control to study the optimal dividend policy; see Asmussen and Taksar (1997), Gerber and Shiu (2004), Gerber and Shiu (2006), Kulenko and Schimidli (2008), Yao et al. (2011) and Jin et al. (2013b). Moreover, to protect insurance companies against the impact on various risks, reinsurance is a standard tool with the goal of reducing and eliminating risk. The primary insurance carrier pays the reinsurance company a certain part of the premiums. In return, the reinsurance company is obliged to share the risk of large claims. Some recent work can be found in Asmusen et al. (2000), Bai and Guo (2008), Bai et al. (2008), Choulli et al. (2001), Pang (2006), Jin et al. (2013a), Wei et al. (2010), Zhang and Siu (2012), Meng and Siu (2011) and references therein. A practitioner manages the reserve and dividend payment against future risks arising from the written CDSs by taking into account reinsurance tools.

The Cramér-Lundberg process (Lundberg (1903)) is inadequate to model the risk and return in our formulation for several reasons. First, classical Cramér-Lundberg process didn't consider the surplus changes coming from the assets, which are held by insurance companies against the liabilities. The assets make income from the investment return and capital gains or losses that are represented in the second term in (2.4). Second, the correlation between the value of the claims against insurers that provide protections for CDSs and the value of the insured securities can't be ignored. When the market value of the insured securities decline, the insurers either compensate the policyholders for the value difference or put up more collateral as requested, both of which will lead to surplus decrease. Hence, the value of the claims are highly negatively correlated with the value of the insured securities. Third, the assets in insurers' portfolio are quite closely correlated to the insured securities. The dependence will increase complexity of the formulation in our problem. In addition, unlike the classical ruin problem or the Cramér-Lundberg approach, our criterion does not focus solely upon the probability of ruin. The criterion in our problem is to maximize the expectation of the discounted value of the utility of dividend until financial ruin under optimal liabilities and dividend strategies.

In this study, we choose different criteria and take into account the risk aversion level for different types of insurers to find the optimal capital requirement or leverage that balances risk against expected growth and return. The value function considered in this stochastic control problem has two variables, which represent the surplus and claim rate. The two control variables are debt ratio and dividend payment rate, respectively. By dynamic programming principle, the value function obeys a second order nonlinear partial differential equation (PDE) generally. Due to the nonlinearity, explicit solutions are generally not able to be obtained for this type of PDE. Fleming and Pang (2004) introduced a subsolution-supersolution method to obtain existence of classical solutions of the Hamilton-Jacobi-Bellman (HJB) equation. In our formulation, the stochastic control problem can be solved analytically. Under general assumptions, we prove the existence of classical solution. Moreover, we obtain the explicit form of the value function and corresponding optimal strategies in some special cases. An example is provided to illustrate the ideas and methodologies. The im- 
pact of reinsurance strategies on the debt management and dividend payment policies are clearly obtained from the analytical solutions of optimal controls.

The rest of the paper is organized as follows. A general formulation of asset value, debt, surplus, insurance liabilities, claim rates, dividend strategies, and assumptions are presented in Section 2. Section 3 deals with optimal debt ratio and dividend payment strategies in logarithm utilities. The subsolution-supersolution method are introduced, and the existence of classical solution of HJB equation is proved in Section 3.1. The verification theorem of optimal value function is presented in Section 3.2. Section 4 deals with optimal debt ratio and dividend payment strategies in power utilities. An example is provided in Section 5 and the impact of reinsurance strategies is considered. Finally, additional remarks are provided in Section 6.

\section{Formulation}

For a large insurer, the surplus process $X(t)$ is described as the difference between the asset value $K(t)$ and liabilities $L(t)$. That is,

$$
X(t)=K(t)-L(t) .
$$

In addition, when the insurer incurs a liability at time $t$, he receives a premium for the amount insured. The collected premium will increase assets and surplus at time $t$. Denote by $\alpha$ be the premium rate, which represents the cost of protection per dollar of insurance liabilities. The asset value increases from the insurance sales during the time period $[t, t+d t]$ is denoted as $\alpha L(t) d t$.

To protect insurance companies against the impact of claim volatilities, reinsurance is a standard tool with the goal of reducing and eliminating risk. The primary insurance carrier pays the reinsurance company a certain part of the premiums. In return, the reinsurance company is obliged to share the risk of large claims. We assume that proportional reinsurance is adopted by the primary insurance company in our model. Within this scheme, the reinsurance company covers a fixed percentage of losses. Let $\lambda$ be an exogenous retention level for the reinsurance policy. Note that $\lambda \in[0,1]$. Denote by $h(\lambda)$ be reinsurance charge rate (the cost of reinsurance protection per dollar of reinsured liabilities) for hedging the adverse claims due to downside risk of the securities' values. From a practical view of point, the cost of reinsurance protection per dollar of reinsured liabilities should be nonnegative and less than 1 . Thus, we assume that $h(\lambda)$ is bounded and $h(\lambda) \in[0,1]$. Hence, the reinsurance charge during the time period $[t, t+d t]$ is denoted as $h(\lambda) L(t) d t$, and only $\lambda L(t) d t$ will be covered by the primary insurance company.

At this premium rate $\alpha$ and reinsurance retention level $\lambda$, there is an elastic demand for insurance contract and the insurer decides how much insurance $L(t)$ to offer at that premium rate and reinsurance retention level. One natural control variable of the insurance company is its liability, the insurance policies sold such as CDS. Let $\pi(t)=L(t) / X(t)$ be the debt ratio of the insurance company. Then, the leverage, which is described as the ratio between asset values and surplus, can be written as $K(t) / X(t)=1+\pi(t)$. To avoid the insurance liabilities being too large, the insurers will decide the optimal liabilities to manage the sale of insurance policies.

We assume that the asset value $K(t)$ in the financial market follows a geometric Brownian Motion process

$$
\frac{d K(t)}{K(t)}=\mu(t) d t+\sigma_{1} d W_{1}(t)
$$

where $\mu(t)$ is the varying drift of the asset and $\sigma_{1}$ is the corresponding volatility and $W_{1}(t)$ is a standard Brownian motion. Particularly, we assume that $\mu(t)$ satisfies the polynomial growth 
condition. That is,

$$
\forall \mu(t) \in C(\mathbb{R}), \mu(t) \leq \theta_{1}\left(1+|t|^{\phi_{1}}\right),
$$

where $\theta_{1}$ and $\phi_{1}$ are positive constants. Hence, combining $(2.1)-(2.2)$, the surplus process in the absence of claims and dividend payment can be denoted by $\widetilde{X}(t)$ and follows

$$
d \widetilde{X}(t)=(\alpha-h(\lambda)) L(t) d t+K(t)\left(\mu(t) d t+\sigma_{1} d W_{1}(t)\right)
$$

We further consider the future claims, which are against insurer's liabilities incurred earlier. The future claims are the required payments to the insured holders of CDS, due to either defaults of the obligor or for collateral calls when the prices of the insured securities decline; see Stein (2012). Surplus declines by the amount of future claims. Denoted by $S(t)$ the future claims up to time $t$. Then we assume that the claims are proportional to the amount of insurance liabilities $L(t)$. Hence, the accumulated claims up to time $T$ is denoted as

$$
S(T)=\int_{0}^{T} c(t) L(t) d t
$$

where $c(t)$ can be considered as a claim rate against liabilities. Since the value of the claims are highly negatively correlated with the value of the insured securities, which is also closely correlated with the assets in insurers' portfolio, the claim rate $c(t)$ is negative correlated to the asset values $K(t)$.

We assume that $c(t)$ is risky and can be described as a stochastic process. It is affected by a series of economic factors such as credit ratings of banks and insurance companies, government regulation, and demand of CDOs in the market, etc. In addition, it is largely influenced by the randomness of economic environment that are described as random shocks. That is, the claim rate $c(t)$ follows a diffusion process

$$
\left\{\begin{array}{l}
d c(t)=g(c(t)) d t+\sigma_{2} d W_{2}(t) \\
c(0)=c
\end{array}\right.
$$

where $g(c(t)): \mathbb{R} \rightarrow \mathbb{R}$ is expected claim rate. $\sigma_{2}$ represents the volatility of the claim rate with $\sigma_{2}>0$. Since the claim rate is negatively correlated to the asset values, we use $-1 \leq \rho<0$ to represent instantaneous correlation between the growth rate of asset value and future claims. Let $W_{2}(t)$ be a standard Brownian motion that is negatively correlated with $W_{1}(t)$ (with a correlation coefficient $\rho$ ). Denote by $\operatorname{Cov}(\cdot, \cdot)$ the covariance between two random variables. Then,

$$
\operatorname{Cov}\left(\frac{d K(t)}{K(t)}, d c(t)\right)=\rho \sigma_{1} \sigma_{2} d t .
$$

We assume that $g(c)$ has bounded derivatives. That is,

$$
\forall g(c) \in C(\mathbb{R}),-\kappa_{3}<g_{c}(c)<\kappa_{2},
$$

where $\kappa_{2}$ and $\kappa_{3}$ are positive constants, $g_{c}$ denote the first derivative of $g$.

Remark 2.1. Note that our formulation as stated in (2.5) and (2.6) can be considered as an extension of equation (6.4) in Stein's work Stein (2012). The solution of (2.6) can be negative. We can interpret $c(t)$ as the sum of the claim rate and the random part of the premium rate (the 
premium rate $\alpha$ is the average of the premium rate). If $c(t)$ is negative, it is because the premium fluctuation.

We are now working on a filtered probability space $\left(\Omega, \mathcal{F},\left\{\mathcal{F}_{t}\right\}, P\right)$, where $\mathcal{F}_{t}$ is the $\sigma$-algebra generated by $\left\{W_{1}(s), W_{2}(s): 0 \leq s \leq t\right\}$ and $\left\{\mathcal{F}_{t}\right\}$ is the filtration satisfying the usual conditions. A dividend strategy $D(\cdot)$ is an $\mathcal{F}_{t}$-adapted process $\{D(t): t \geq 0\}$ corresponding to the accumulated amount of dividends paid up to time $t$ such that $D(t)$ is a nonnegative and nondecreasing stochastic process that is right continuous and have left limits with $D\left(0^{-}\right)=0$. In this paper, we consider the optimal dividend strategy where the dividend payments are proportional to the surplus with a dividend payment rate $z(t)$. Denote $\Gamma=[0, M], 0<M<\infty$. As a result, we write $D(t)$ as

$$
d D(t)=z(t) X(t) d t
$$

where $z(t)$ is an $\mathcal{F}_{t}$-adapted process and $0 \leq z(t) \leq M$. Thus, taking into consider the impact of reinsurance, the insurer's surplus process in the presence of claims and dividend payments is given by

$$
d X(t)=d \widetilde{X}(t)-\lambda d S(t)-d D(t) .
$$

Together with the initial condition, (2.9) follows

$$
\left\{\begin{array}{l}
d X(t)=[(\alpha-h(\lambda)-\lambda c(t)) L(t)+\mu(t) K(t)-z(t) X(t)] d t+K(t) \sigma_{1} d W_{1}(t), \\
X(0)=x \geq 0
\end{array}\right.
$$

for all $t<\tau$ and we impose $X(t)=0$ for all $t>\tau$, where $\tau=\inf \{t \geq 0: X(t)<0\}$ represents the time of financial ruin. Suppose the optimal payout strategy is applied subsequently.

Recall that $\pi(t)$ represents the debt ratio, (2.10) can be written as

$$
\left\{\begin{array}{l}
\frac{d X(t)}{X(t)}=[\pi(t)(\alpha-h(\lambda)-\lambda c(t)+\mu(t))+\mu(t)-z(t)] d t+(\pi(t)+1) \sigma_{1} d W_{1}(t), \\
X(0)=x
\end{array}\right.
$$

For debt ratio $\pi(t)$, we assume that $\forall T \in(0, \infty)$,

$$
E \int_{0}^{T} \pi^{2}(t) d t<\infty
$$

For dividend payment rate $z(t)$, we assume $z(t)$ is non-negative and subject to an upper bound. A strategy $u(\cdot)=\{(\pi(t), z(t)): t \geq 0\}$ being progressively measurable with respect to $\left\{W_{1}(s), W_{2}(s)\right.$ : $0 \leq s \leq t\}$ is called an admissible strategy. Denote the collection of all admissible strategies or admissible controls by $\mathcal{A}$. Then the admissible strategy set $\mathcal{A}$ can be defined as

$$
\mathcal{A}=\left\{u(t)=(\pi(t), z(t)) \in \mathbb{R} \times \mathbb{R}: E \int_{0}^{T} \pi^{2}(t) d t<\infty ; 0 \leq z(t) \leq M<\infty\right\} .
$$

The representative financial institute is risk averse and the objective is to maximize the expectation of the discounted value of the utility of dividend until financial ruin. Denote by $r>0$ the discount factor. For an arbitrary admissible pair $u=(\pi, z)$, the performance function is the expected discounted dividend until ruin, and is given by

$$
J(x, c, u(\cdot))=E_{x, c}\left[\int_{0}^{\tau} e^{-r t} U(z(t) X(t)) d t\right],
$$


where $E_{x, c}$ denotes the expectation conditioned on $X(0)=x$ and $c(0)=c$.

We are interested in finding the optimal dividend payment rate and debt ratio to maximize the performance function $J(x, c, u(\cdot))$. Define $V(x, c)$ as the optimal value of the corresponding problem. That is,

$$
V(x, c)=\sup _{u(\cdot) \in \mathcal{A}} J(x, c, u(\cdot)) .
$$

Setting $u(t)$ to be any quantity such that it does not change the value of $V(x(\tau), c(\tau))$ for $t \geq \tau$, that is, $z(t)=0$ for $t \geq \tau$, Therefore, (2.14) can be rewritten as

$$
J(x, c, u(\cdot))=E_{x, c}\left[\int_{0}^{\infty} e^{-r t} U(z(t) X(t)) d t\right] .
$$

To solve a stochastic control problem, one usually uses a dynamic programming approach. This in turn requires considering the generator (an operator) of the controlled process involved and use it to derive a partial differential equation, known as HJB equation, satisfied by the value function. The solution of the HJB equation then yields the optimal control and optimal value. assuming the existence of optimal control, for an arbitrary $V(\cdot, \cdot) \in C^{2}(\mathbb{R} \times \mathbb{R})$, define an operator $\mathcal{L}^{u}$ by

$$
\begin{aligned}
\mathcal{L}^{u} V(x, c)=\frac{1}{2} & V_{x x} \sigma_{1}^{2}(\pi+1)^{2} x^{2}+\frac{1}{2} \sigma_{2}^{2} V_{c c}+V_{x c} \rho \sigma_{1} \sigma_{2} x(\pi+1) \\
& +V_{x}(\pi(\alpha-h(\lambda)-\lambda c+\mu)+\mu-z) x+g(c) V_{c}
\end{aligned}
$$

where $V_{x}, V_{c}, V_{x x}$, and $V_{c c}$ denote the first-order and the second-order partial derivatives with respect to $x$ and $c$, respectively. Formally, the value function (2.15) satisfies the Hamilton-JacobiBellman equation

$$
\max _{u}\left\{\mathcal{L}^{u} V(x, c)-r V(x, c)+U(z x)\right\}=0 .
$$

Using $\pi$ and $c$ to represent the controls, (2.18) can be rewritten as

$$
\begin{gathered}
\max _{\pi}\left[\frac{1}{2} V_{x x} \sigma_{1}^{2}(\pi+1)^{2} x^{2}+V_{x c} \rho \sigma_{1} \sigma_{2} x(\pi+1)+V_{x} x \pi(\alpha-h(\lambda)-\lambda c+\mu)\right] \\
+\max _{z}\left[-z x V_{x}+U(z x)\right]+\frac{1}{2} \sigma_{2}^{2} V_{c c}+\mu x V_{x}+g(c) V_{c}-r V(x, c)=0 .
\end{gathered}
$$

\section{Case 1: Logarithm Utility Function}

There are some utility functions that can be selected as the objective function in the optimization process. We will consider two major types of utility functions: the logarithm utility and power utility. Each type of the utility function is adopted by the practitioners based on their specific return and risk objectives. In the logarithm utility case, the decision makers tend to generate a high dividend payment under the constraint of default risks. Moreover, the concave utility function shows that extremely heavy penalty will be placed on a debt that would lead to zero or low dividend payments. As the power utility function has a constant relative risk aversion, the power utility function shows its advantages in describing the risk aversion level of the decision makers. The power utility function case will be analyzed in Section 4.

\subsection{Optimal Controls and Value Function}

We construct a solution of (2.19) with the form

$$
V(x, c)=a \ln x+Y(c) .
$$


With appropriate values of $a$ and $Y(c),(3.1)$ will be verified to be the solution of (2.19) in Section 3.2. To determine $a$ and $Y(c)$, we plug (3.1) into (2.19). Then we have

$$
\begin{aligned}
\max _{\pi}\left[-\frac{a}{2} \sigma_{1}^{2} \pi^{2}+\pi a(\alpha-h(\lambda)\right. & \left.\left.-\lambda c+\mu-\sigma_{1}^{2}\right)\right]+\max _{z}[-a z+\ln z]+(1-a r) \ln x \\
& +\frac{1}{2} \sigma_{2}^{2} Y_{c c}(c)+g(c) Y_{c}(c)-r Y(c)-\frac{a}{2} \sigma_{1}^{2}+a \mu=0 .
\end{aligned}
$$

Since (3.2) holds for all $x$, we have

$$
a=\frac{1}{r}
$$

and

$$
V(x, c)=\frac{1}{r} \ln x+Y(c) .
$$

In view of $(3.2)$, the optimal debt ratio $\pi^{*}$ is obtained as

$$
\pi^{*}=\frac{\alpha-h(\lambda)-\lambda c+\mu-\sigma_{1}^{2}}{\sigma_{1}^{2}},
$$

and the optimal dividend payment rate follows

$$
z^{*}=r
$$

Substituting the optimal controls into (3.2), it yields that

$$
\frac{1}{2} \sigma_{2}^{2} Y_{c c}(c)+g(c) Y_{c}(c)-r Y(c)+N(c)=0,
$$

where

$$
N(c)=\frac{\left(\alpha-h(\lambda)-\lambda c+\mu-\sigma_{1}^{2}\right)^{2}}{2 r \sigma_{1}^{2}}+\ln r-1-\frac{1}{2 r} \sigma_{1}^{2}+\frac{\mu}{r} .
$$

Let $\tilde{Y}(c)$ be a classical solution of $(3.7)$, then we will verify that the proposed value function

$$
\widetilde{V}(x, c)=\frac{1}{r} \ln x+\widetilde{Y}(c)
$$

equals the value function $V(x, c)$ defined in (2.15).

To obtain the classical solution of (3.7), we use the subsolution and supersolution method in Fleming and Pang (2004). The subsolution and supsolution will be defined as follows.

Definition 3.1. A solution $Y_{1}(c)$ is said to be a subsolution of (3.7) iff $\forall c \in \mathbb{R}, Y_{1}(c) \in C^{2}(\mathbb{R})$ and $Y_{1}(c)$ satisfies

$$
\frac{1}{2} \sigma_{2}^{2} Y_{c c}(c)+g(c) Y_{c}(c)-r Y(c)+N(c) \geq 0 .
$$

A solution $Y_{2}(c)$ is said to be a supersolution of $(3.7)$ iff $\forall c \in \mathbb{R}, Y_{2}(c) \in C^{2}(\mathbb{R})$ and $Y_{2}(c)$ satisfies

$$
\frac{1}{2} \sigma_{2}^{2} Y_{c c}(c)+g(c) Y_{c}(c)-r Y(c)+N(c) \leq 0 .
$$

Moreover, if $\forall c \in \mathbb{R}$

$$
Y_{1}(c) \leq Y_{2}(c),
$$

we say $Y_{1}(c)$ and $Y_{2}(c)$ are an ordered pair of subsolution and supersolution. 
Let $f(Y, c)=-r Y(c)+N(c)$, then (3.9) and (3.10) can be rewritten as

$$
\frac{1}{2} \sigma_{2}^{2} Y_{c c}(c)+g(c) Y_{c}(c)+f(Y, c) \geq 0
$$

and

$$
\frac{1}{2} \sigma_{2}^{2} Y_{c c}(c)+g(c) Y_{c}(c)+f(Y, c) \leq 0
$$

To proceed, we will first find an ordered pair of subsolution and supersolution $\left(Y_{1}(c), Y_{2}(c)\right)$. Then we can prove the existence of a classical solution $\widetilde{Y}(c)$ of $(3.7)$.

Lemma 3.2. Let

$$
\tilde{y}=\frac{1}{r}\left(\ln r-1-\frac{1}{2 r} \sigma_{1}^{2}+\frac{\mu}{r}\right) .
$$

Then $\tilde{y}$ is a subsolution of (3.7). Moreover, $\forall c \in \mathbb{R}, f(\tilde{y}, c) \geq 0$.

Proof. Since $\tilde{y}$ is constant,

$$
\frac{1}{2} \sigma_{2}^{2} \tilde{y}_{c c}+g(c) \tilde{y}_{c}=0 .
$$

To verify that $\tilde{y}$ is a subsolution of $(3.7)$, it is sufficient to verify $f(\tilde{y}, c)>0, \forall c \in \mathbb{R}$. In view of (3.7), we have

$$
f(\tilde{y}, c)=\frac{\left(\alpha-h(\lambda)-\lambda c+\mu-\sigma_{1}^{2}\right)^{2}}{2 r \sigma_{1}^{2}} .
$$

Then,

$$
\frac{1}{2} \sigma_{2}^{2} \tilde{y}_{c c}(c)+g(c) \tilde{y}_{c}(c)+f(\tilde{y}, c) \geq 0
$$

Hence, $\tilde{y}$ is a subsolution of $(3.7)$, and $f(\tilde{y}, c) \geq 0, \forall c \in \mathbb{R}$.

Lemma 3.3. Let

$$
\widehat{y}(c)=\frac{\left(\alpha-h(\lambda)-\lambda c+\mu-\sigma_{1}^{2}\right)^{2}}{r^{2} \sigma_{1}^{2}}+\frac{1}{r}\left(\frac{\lambda^{2} \sigma_{2}^{2}}{r^{2} \sigma_{1}^{2}}+\ln r-\frac{1}{2 r} \sigma_{1}^{2}+\frac{\mu}{r}+\widetilde{Q}\right),
$$

where $\widetilde{Q}$ is a sufficient large positive constant such that

$$
\widetilde{Q}>\frac{2 \lambda^{2}\left(g(0)+c \kappa_{2}\right)^{2}}{r^{2} \sigma_{1}^{2}} .
$$

Then $\widehat{y}(c)>\tilde{y}(c)$, and $\widehat{y}(c)$ is a supersolution of $(3.7)$.

Proof. In view of the definition of $\widehat{y}(c)$ and $\tilde{y}(c)$, we have

$$
\widehat{y}(c)-\tilde{y}(c)>\frac{\left(\alpha-h(\lambda)-\lambda c+\mu-\sigma_{1}^{2}\right)^{2}}{r^{2} \sigma_{1}^{2}}+\frac{2\left(g(0)+c \kappa_{2}\right)^{2}+\lambda^{2} \sigma_{2}^{2}}{r^{3} \sigma_{1}^{2}}+\frac{1}{r} .
$$

Hence, $\widehat{y}(c)>\tilde{y}(c)$ as $r>0$. On the other hand, $\widehat{y}(c)$ is a quadratic function of $c$, we have

$$
\widehat{y}_{c c}(c)=\frac{2 \lambda^{2}}{r^{2} \sigma_{1}^{2}} \text { and } \widehat{y}_{c}(c)=\frac{2 \lambda}{r^{2} \sigma_{1}^{2}}\left(\alpha-h(\lambda)-\lambda c+\mu-\sigma_{1}^{2}\right)
$$


Moreover,

$$
\begin{aligned}
f(\widehat{y}, c) \\
=-r \widehat{y}(c)+N(c) \\
=-\frac{\left(\alpha-h(\lambda)-\lambda c+\mu-\sigma_{1}^{2}\right)^{2}}{r \sigma_{1}^{2}}-\left(\frac{\lambda^{2} \sigma_{2}^{2}}{r^{2} \sigma_{1}^{2}}+\ln r-\frac{1}{2 r} \sigma_{1}^{2}+\frac{\mu}{r}+\widetilde{Q}\right) \\
\quad+\frac{\left(\alpha-h(\lambda)-\lambda c+\mu-\sigma_{1}^{2}\right)^{2}}{2 r \sigma_{1}^{2}}+\ln r-1-\frac{1}{2 r} \sigma_{1}^{2}+\frac{\mu}{r} \\
=-\frac{\left(\alpha-h(\lambda)-\lambda c+\mu-\sigma_{1}^{2}\right)^{2}}{2 r \sigma_{1}^{2}}-\frac{\lambda^{2} \sigma_{2}^{2}}{r^{2} \sigma_{1}^{2}}-\widetilde{Q}-1 .
\end{aligned}
$$

In view of (2.7), there exists a $\xi \in[0, c]$ such that

$$
g(c)=g(0)+c g_{c}(\xi) \leq g(0)+c \kappa_{2} .
$$

Hence, combining the equations (3.15) and (3.16), the left sides of (3.12) follows

$$
\begin{aligned}
& \frac{1}{2} \sigma_{2}^{2} \widehat{y}_{c c}(c)+g(c) \widehat{y}_{c}(c)+f(\widehat{y}, c) \\
& =\frac{2 \lambda g(c)}{r^{2} \sigma_{1}^{2}}\left(\alpha-h(\lambda)-\lambda c+\mu-\sigma_{1}^{2}\right)-\frac{\left(\alpha-h(\lambda)-\lambda c+\mu-\sigma_{1}^{2}\right)^{2}}{2 r^{2} \sigma_{1}^{2}}-\widetilde{Q}-1 \\
& \leq-\frac{1}{2 r^{2} \sigma_{1}^{2}}\left[\left(\alpha-h(\lambda)-\lambda c+\mu-\sigma_{1}^{2}\right)-2 \lambda\left(g(0)+c \kappa_{2}\right)\right]^{2}-1 \\
& \leq 0 .
\end{aligned}
$$

Therefore, $\widehat{y}(c)$ is a supersolution of $(3.7)$.

Theorem 3.4. There exists a classical solution of equation (3.7) denoted by $\widetilde{Y}(c)$ such that

$$
\tilde{y} \leq \tilde{Y}(c) \leq \widehat{y}(c),
$$

where $\tilde{y}$ and $\widehat{y}(c)$ are defined in (3.13) and (3.14), respectively.

Proof. In accordance with the Lemma 3.2 and Lemma 3.3, an ordered pair of subsolution and supersolution of equation (3.7) are obtained. The existence of a classical solution can be proved by Theorem 5.2 in Chapter 7 in Pao (1992).

\subsection{Verification Theorem}

In this section, we will focus on the verification theorem. To begin with, we will provide a lemma that will be used in verification theorem.

Lemma 3.5. Assume that (2.7) is satisfied, then (2.6) has a unique strong solution. For simplicity, denote by $c(t)$ the strong solution, then we have

$$
E|c(t)|^{2} \leq \beta_{1} e^{\beta_{2} t},
$$

where $\beta_{1}$ and $\beta_{2}$ are positive constants which are independent of $t$. 
Proof. By virtue of Theorem 5.2.1 in Chapter 5 of Øksendal (2003), it is not hard to verify the existence and uniqueness result about strong solution of (2.6) under the condition of (2.7). Moreover, using Itô lemma, we obtain

$$
\begin{aligned}
d c(t)^{2} & =2 c(t) d c(t)+(d c(t))^{2} \\
& =\left(2 c(t) g(c)+\sigma_{2}^{2}\right) d t+2 c(t) \sigma_{2} d W_{2} .
\end{aligned}
$$

Hence, we have

$$
c(t)^{2}=c^{2}+\int\left(2 c(t) g(c)+\sigma_{2}^{2}\right) d t+\widetilde{M}(t)
$$

where

$$
\widetilde{M}(t)=\int 2 c(t) \sigma_{2} d W_{2}
$$

Note that $\widetilde{M}(t)$ is a martingale. Define $\tilde{\mu}(t)$ as the second moment of $c(t)$. That is,

$$
\tilde{\mu}(t)=E\left[c^{2}(t)\right] .
$$

Then, we obtain

$$
\left.\tilde{\mu}(t)=c^{2}+\int E\left[2 c(t) g(c)+\sigma_{2}^{2}\right)\right] d t
$$

In view of $(2.7)$, we have

$$
g(c) \leq g(0)+\kappa_{2} c(t)
$$

Hence, (3.19) can be rewritten as

$$
\begin{aligned}
\tilde{\mu}(t) & \leq c^{2}+\int E\left[2 c(t)\left(g(0)+\kappa_{2} c(t)\right)+\sigma_{2}^{2}\right] d t \\
& =c^{2}+\sigma_{2}^{2} t+2 \kappa_{2} \int E\left[c^{2}(t)\right] d t+\int E[2 c(t) g(0)] d t .
\end{aligned}
$$

By using Cauchy-Schwarz inequality, for arbitrary small $\varepsilon>0,(3.20)$ can be rewritten as

$$
\begin{aligned}
\frac{d \tilde{\mu}(t)}{d t} & \left.=E\left[2 c(t) g(c)+\sigma_{2}^{2}\right)\right] \\
& \leq \sigma_{2}^{2}+2 \kappa_{2} E\left[c^{2}(t)\right]+\varepsilon E\left[c^{2}(t)\right]+\frac{g^{2}(0)}{\varepsilon} \\
& =\left(2 \kappa_{2}+\varepsilon\right) \tilde{\mu}(t)+\sigma_{2}^{2}+\frac{g^{2}(0)}{\varepsilon} .
\end{aligned}
$$

Let

$$
\widetilde{K}=\sigma_{2}^{2}+\frac{g^{2}(0)}{\varepsilon} .
$$

Note that $\widetilde{K}$ is a positive constant since $\sigma_{2}>0$. Therefore,

$$
\begin{aligned}
e^{-\left(2 \kappa_{2}+\varepsilon\right) t} \tilde{\mu}(t) & =\tilde{\mu}(0)+\int_{0}^{t} \widetilde{K} e^{-\left(2 \kappa_{2}+\varepsilon\right) s} d s \\
& =\tilde{\mu}(0)+\frac{\widetilde{K}}{2 \kappa_{2}+\varepsilon}\left(1-e^{-\left(2 \kappa_{2}+\varepsilon\right) t}\right) .
\end{aligned}
$$


Note that $\tilde{\mu}(0)=c^{2}$, then we have

$$
\begin{aligned}
\tilde{\mu}(t) & \leq c^{2} e^{\left(2 \kappa_{2}+\varepsilon\right) t}+\frac{\widetilde{K}\left(e^{\left(2 \kappa_{2}+\varepsilon\right) t}-1\right)}{2 \kappa_{2}+\varepsilon} \\
& =\left(c^{2}+\frac{\widetilde{K}}{2 \kappa_{2}+\varepsilon}\right) e^{\left(2 \kappa_{2}+\varepsilon\right) t}-\frac{\widetilde{K}}{2 \kappa_{2}+\varepsilon} \\
& \leq\left(c^{2}+\frac{\widetilde{K}}{2 \kappa_{2}+\varepsilon}\right) e^{\left(2 \kappa_{2}+\varepsilon\right) t} .
\end{aligned}
$$

Hence, we obtain

$$
E|c(t)|^{2} \leq \beta_{1} e^{\beta_{2} t}
$$

where

$$
\begin{aligned}
& \beta_{1}=c^{2}+\frac{\widetilde{K}}{2 \kappa_{2}+\varepsilon}, \\
& \beta_{2}=2 \kappa_{2}+\varepsilon
\end{aligned}
$$

and $\beta_{1}$ and $\beta_{2}$ are positive constants.

Now we are in a position to establish the verification theorem for the optimal debt ratio and dividend policies.

Theorem 3.6. Suppose there exists a function $\widetilde{Y}(t)$ such that $(3.17)$ holds, and that $\tilde{Y}(t)$ solves (3.7). Assume that $r>2 \kappa_{2}$. Let

$$
\widetilde{V}(x, c)=\frac{1}{r} \ln x+\widetilde{Y}(c) .
$$

Then,

(a) For all admissible pairs of control policies $u=(\pi, z) \in \mathcal{A}$,

$$
\widetilde{V}(x, c) \geq J(x, c, u)=E_{x, c} \int_{0}^{\infty} e^{-r t} \ln (z(t) X(t)) d t .
$$

(b) If $u^{*}=\left(\pi^{*}, z^{*}\right)$ satisfies the following:

$$
\begin{aligned}
& \pi^{*}=\frac{\alpha-h(\lambda)-\lambda c+\mu-\sigma_{1}^{2}}{\sigma_{1}^{2}}, \\
& c^{*}=r .
\end{aligned}
$$

Then $u^{*} \in \mathcal{A}$. We have

$$
\widetilde{V}(x, c) \geq J\left(x, c, u^{*}\right)=E_{x, c} \int_{0}^{\infty} e^{-r t} \ln \left(z^{*}(t) X(t)\right) d t .
$$

Moreover, $\widetilde{V}(x, c)$ is the value function defined in $(2.15)$. That is, $\widetilde{V}(x, c)=V(x, c)$.

Proof. Applying Itô's lemma to $\widetilde{V}(X(t), b(t))$, we obtain

$$
\begin{aligned}
d \widetilde{V}(X(t), c(t)) & =\widetilde{V}_{x} d X(t)+\widetilde{V}_{c} d c(t)+\frac{1}{2} \widetilde{V}_{x x}(d X(t))^{2}+\frac{1}{2} \widetilde{V}_{c c}(d c(t))^{2}+\widetilde{V}_{x c} d X(t) d c(t) \\
& =\mathcal{L}^{u} \widetilde{V}(X(t), c(t)) d t+(\pi(t)+1) \sigma_{1} \widetilde{V}_{x} d W_{1}+\sigma_{2} \widetilde{V}_{c} d W_{2}
\end{aligned}
$$


Then,

$$
\widetilde{V}(X(t), c(t))-\widetilde{V}(x, c)=\int_{0}^{t} \mathcal{L}^{u} \widetilde{V}(X(s), c(s)) d s+\bar{M}_{1}+\bar{M}_{2}
$$

where

$$
\begin{aligned}
& \bar{M}_{1}=\int_{0}^{t}(\pi(t)+1) \sigma_{1} \widetilde{V}_{x} d W_{1}, \\
& \bar{M}_{2}=\int_{0}^{t} \sigma_{2} \widetilde{V}_{c} d W_{2} .
\end{aligned}
$$

Note that $\bar{M}_{1}$ and $\bar{M}_{2}$ are martingales. Similarly, applying Itô's lemma to $e^{-r t} \widetilde{V}(x, c)$, we have

$$
\begin{aligned}
d\left(e^{-r t} \widetilde{V}(X(t), c(t))\right) & =e^{-r t} d \widetilde{V}(X(t), c(t))-r e^{-r t} \widetilde{V}(X(t), c(t)) d t \\
& =e^{-r t}\left[\left(\mathcal{L}^{u} \widetilde{V}(X(t), c(t))-r \widetilde{V}(X(t), c(t))\right) d t+d\left(\bar{M}_{1}+\bar{M}_{2}\right)\right]
\end{aligned}
$$

In view of (2.18), for any control $u \in \mathcal{A}$, we have

$$
\mathcal{L}^{u} \widetilde{V}(X(t), c(t))-r \widetilde{V}(X(t), c(t)) \leq-\ln (z(t) X(t)) .
$$

Integrating (3.28) in $[0, T]$ and taking expectation on both sides, then applying the above inequality, we have

$$
E \int_{0}^{T} d\left(e^{-r t} \widetilde{V}(X(t), c(t))\right) \leq-E \int_{0}^{T} e^{-r t} \ln (z(t) X(t)) d t
$$

Then,

$$
E e^{-r T} \widetilde{V}(X(T), c(T))-\widetilde{V}(x, c) \leq-E \int_{0}^{T} e^{-r t} \ln (z(t) X(t)) d t
$$

Hence,

$$
\begin{aligned}
\widetilde{V}(x, c) & \geq E \int_{0}^{T} e^{-r t} \ln (z(t) X(t)) d t+E e^{-r T} \widetilde{V}(X(T), c(T)) \\
& =E \int_{0}^{T} e^{-r t} \ln (z(t) X(t)) d t+E e^{-r T}\left[\frac{1}{r} \ln X(T)+\widetilde{Y}(c(T))\right] .
\end{aligned}
$$

To verify (3.25), we need to show that

$$
\limsup _{T \rightarrow \infty} E e^{-r T} \tilde{V}(X(T), c(T)) \geq 0 .
$$

Since $\tilde{Y}(c)$ is bounded with subsolution $\tilde{y}$ and supersolution $\widehat{y}(c)$ in $(3.17)$, where $\tilde{y}$ is independent of $T$. We have

$$
\limsup _{T \rightarrow \infty} E e^{-r T} \tilde{Y}(c(T)) \geq 0 .
$$

Hence, it is sufficient to show that

$$
\limsup _{T \rightarrow \infty} E e^{-r T} \ln X(T) \geq 0 .
$$

Applying Itô's lemma to $\ln X(t)$, we have

$$
d \ln X(t)=\left[\pi(t)(\alpha-h(\lambda)-\lambda c(t)+\mu(t))+\mu(t)-z(t)-\frac{1}{2} \sigma_{1}^{2}(\pi(t)+1)^{2}\right] d t+(\pi(t)+1) \sigma_{1} d W_{1} .
$$


Hence,

$$
\begin{aligned}
& E e^{-r T} \ln X(T) \\
& =e^{-r T} E \int_{0}^{T}\left[\pi(t)(\alpha-h(\lambda)-\lambda c(t)+\mu(t))+\mu(t)-z(t)-\frac{1}{2} \sigma_{1}^{2}(\pi(t)+1)^{2}\right] d t+e^{-r T} \ln x .
\end{aligned}
$$

where

$$
\begin{aligned}
& A_{1}=-\frac{1}{2} \sigma_{1}^{2} \pi^{2}(t), \\
& A_{2}=-\lambda c(t) \pi(t), \\
& A_{3}=\pi(t)\left(\mu(t)-\sigma_{1}^{2}\right), \\
& A_{4}=-z(t) \\
& A_{5}=\pi(t)(\alpha-h(\lambda)), \\
& A_{6}=\mu(t)-\frac{1}{2} \sigma_{1}^{2} .
\end{aligned}
$$

To proceed, we will prove

$$
\limsup _{T \rightarrow \infty} e^{-r T} E \int_{0}^{T} A_{i} d t \geq 0, \quad \forall i=1, \ldots, 6 .
$$

The definition of admissible strategies yields that

$$
\begin{aligned}
\limsup _{T \rightarrow \infty} e^{-r T} E \int_{0}^{T} A_{1} d t & \geq \lim _{T \rightarrow \infty}-\frac{\sigma_{1}^{2}}{2} e^{-r T} E \int_{0}^{T} \pi^{2}(t) d t \\
& =0 .
\end{aligned}
$$

By virtue of Lemma 3.5, if $r>2 \kappa_{2}$, we have

$$
\lim _{T \rightarrow \infty} e^{-r T} E \int_{0}^{T} \lambda^{2} c^{2}(t) d t=0 .
$$

The Cauchy-Schwarz inequality then leads to

$$
\begin{aligned}
\limsup _{T \rightarrow \infty} e^{-r T} E \int_{0}^{T} A_{2} d t & \geq-\lim _{T \rightarrow \infty} \frac{1}{2} e^{-r T} E \int_{0}^{T}\left[c^{2}(t)+\pi^{2}(t)\right] d t \\
& =0 .
\end{aligned}
$$

Referring to (2.3) and (3.37), we have

$$
\begin{aligned}
\limsup _{T \rightarrow \infty} e^{-r T} E \int_{0}^{T} A_{3} d t & \geq-\lim _{T \rightarrow \infty} \frac{1}{2} e^{-r T} E \int_{0}^{T}\left[\left(\mu(t)-\sigma_{1}^{2}\right)^{2}+\pi^{2}(t)\right] d t \\
& =0 .
\end{aligned}
$$

Moreover,

$$
\begin{aligned}
\limsup _{T \rightarrow \infty} e^{-r T} E \int_{0}^{T} A_{4} d t & \geq-\lim _{T \rightarrow \infty} e^{-r T} M T \\
& =0
\end{aligned}
$$


Due to the boundness of $\alpha$ and $h(\lambda)$, we have

$$
\begin{aligned}
\limsup _{T \rightarrow \infty} e^{-r T} E \int_{0}^{T} A_{5} d t & \geq-\lim _{T \rightarrow \infty} \frac{1}{2} e^{-r T} E \int_{0}^{T}\left[(\alpha-h(\lambda))^{2}+\pi^{2}(t)\right] d t \\
& =0,
\end{aligned}
$$

and

$$
\begin{aligned}
\limsup _{T \rightarrow \infty} e^{-r T} E \int_{0}^{T} A_{6} d t & \geq-\lim _{T \rightarrow \infty} \frac{1}{2} e^{-r T} E \int_{0}^{T}\left[-\frac{1}{2} \sigma_{1}^{2}+\frac{1}{2}\left(\mu^{2}(t)+1\right)\right] d t \\
& =-\lim _{T \rightarrow \infty} \frac{1}{2} e^{-r T}\left[-\frac{1}{2}\left(\sigma_{1}^{2}-1\right) T+E \int_{0}^{T} \mu^{2}(t) d t\right] \\
& =0 .
\end{aligned}
$$

Hence, combining (3.37) to (3.42), we obtain

$$
\limsup _{T \rightarrow \infty} E e^{-r T} \ln X(T) \geq 0 .
$$

Therefore, (3.32) is satisfied so (3.25) is verified.

Consider the debt ratio and dividend payment rate strategies $u^{*}=\left(\pi^{*}, z^{*}\right)$,

$$
\begin{aligned}
& \pi^{*}=\frac{\alpha-h(\lambda)-\lambda c+\mu-\sigma_{1}^{2}}{\sigma_{1}^{2}}, \\
& z^{*}=r .
\end{aligned}
$$

It is not hard to show that

$$
\begin{aligned}
& \pi^{*} \in \arg \max _{\pi}\left[\frac{1}{2} V_{x x} \sigma_{1}^{2}(\pi+1)^{2} x^{2}+V_{x c} \rho \sigma_{1} \sigma_{2} x(\pi+1)+V_{x} \pi(\alpha-h(\lambda)-\lambda c+\mu)\right], \\
& z^{*} \in \arg \max _{z}\left[-z x V_{x}+\ln (z x)\right] .
\end{aligned}
$$

Then $u^{*} \in \mathcal{A}$. Let $\left(X^{*}(t), c^{*}(t)\right)$ be the corresponding trajectories of $u^{*}$. We have

$$
\mathcal{L}^{u^{*}} \widetilde{V}\left(X^{*}(t), c^{*}(t)\right)-r \widetilde{V}\left(X^{*}(t), c^{*}(t)\right)=-\ln \left(z^{*}(t) X^{*}(t)\right) .
$$

Hence,

$$
\widetilde{V}(x, c)=E \int_{0}^{T} e^{-r t} \ln \left(z^{*}(t) X^{*}(t)\right) d t+E e^{-r T} \widetilde{V}\left(X^{*}(T), c^{*}(T)\right) .
$$

To prove (3.24), we need only verify

$$
\widetilde{V}(x, c) \leq E \int_{0}^{\infty} e^{-r t} \ln \left(z^{*}(t) X^{*}(t)\right) d t .
$$

That is, it is sufficient to show that

$$
\liminf _{T \rightarrow \infty} E e^{-r T} \widetilde{V}\left(X^{*}(T), c^{*}(T)\right) \leq 0 .
$$

Similar to (3.36),

$$
\begin{aligned}
& E e^{-r T} \ln X^{*}(T) \\
& =e^{-r T} E \int_{0}^{T}\left[\pi^{*}(t)(\alpha-h(\lambda)-\lambda c(t)+\mu(t))+\mu(t)-z^{*}(t)-\frac{1}{2} \sigma_{1}^{2}\left(\pi^{*}(t)+1\right)^{2}\right] d t+e^{-r T} \ln x \\
& \leq e^{-r T}\left\{E \int_{0}^{T}\left(\frac{(\alpha-h(\lambda)-\lambda c(t)+\mu(t))^{2}}{\sigma_{1}^{2}}+\mu(t)\right) d t-r T\right\}+e^{-r T} \ln x .
\end{aligned}
$$


By virtue of the techniques in (3.37) to (3.42), we have

$$
\liminf _{T \rightarrow \infty} E e^{-r T} \ln \left(X^{*}(T)\right) \leq 0 .
$$

Considering the fact that $\widetilde{Y}(c)$ is bounded, we can show that

$$
\liminf _{T \rightarrow \infty} E e^{-r T} \tilde{Y}\left(c^{*}\right) \leq 0 .
$$

Thus, (3.43) is satisfied. Combining with (3.25) and (3.43), we have

$$
V(x, c)=E \int_{0}^{\infty} e^{-r t} \ln \left(z^{*}(t) X^{*}(t)\right) d t
$$

Then $(b)$ is proved.

\section{Case 2: Power Utility Function}

In this section, we consider a power utility function

$$
U(z(t) X(t))=\frac{1}{\gamma}(z(t) X(t))^{\gamma}
$$

where $0<\gamma<1$. Hence, (2.16) can be rewritten as

$$
J(x, c, u(\cdot))=E_{x, c}\left[\int_{0}^{\infty} e^{-r t} \frac{1}{\gamma}(z(t) X(t))^{\gamma} d t\right] .
$$

It is shown that the objective function and value function are homogeneous in $x$ with order $\gamma$. That is,

$$
V(x, c)=\sup _{u(\cdot) \in \mathcal{A}} J(x, c, u(\cdot))=\sup _{u(\cdot) \in \mathcal{A}} x^{\gamma} J(1, c, u(\cdot))=x^{\gamma} V(1, c) .
$$

We can exploit the homogeneity to construct the solution of value function.

\subsection{Optimal Controls and Value Function}

We construct a solution of (2.19) with the form

$$
V(x, c)=\frac{x^{\gamma}}{\gamma} Y(c)
$$

With appropriate values of $Y(c),(4.3)$ will be verified to be the solution of (2.19) in Section 4.2. To determine $Y(c)$, we plug (4.3) into (2.19). Then we have

$$
\begin{aligned}
& 0=\max _{\pi}\left\{\frac{1}{2}(\gamma-1) Y(c) \sigma_{1}^{2} \pi^{2}+\pi\left[Y(c)\left(\alpha-h(\lambda)-\lambda c+\mu+(\gamma-1) \sigma_{1}^{2}\right)+Y_{c}(c) \rho \sigma_{1} \sigma_{2}\right]\right\} \\
& +\max _{z}\left[-z Y(c)+\frac{1}{\gamma} z^{\gamma}\right]+Y(c)\left[\frac{1}{2} \sigma_{1}^{2}(\gamma-1)+\mu-\frac{r}{\gamma}\right]+Y_{c}(c)\left[\rho \sigma_{1} \sigma_{2}+\frac{g(c)}{\gamma}\right]+\frac{1}{2 \gamma} \sigma_{2}^{2} Y_{c c}(c) .
\end{aligned}
$$

In view of (4.4), the optimal debt ratio $\pi^{*}$ is obtained as

$$
\pi^{*}=\frac{\alpha-h(\lambda)-\lambda c+\mu+(\gamma-1) \sigma_{1}^{2}}{(1-\gamma) \sigma_{1}^{2}}+\frac{Y_{c}(c) \rho \sigma_{2}}{(1-\gamma) Y(c) \sigma_{1}},
$$


and the dividend payment rate follows

$$
z^{*}=Y(c)^{\frac{1}{\gamma-1}}
$$

Substituting the optimal controls in (4.4) and multiplying both sides by $Y(c),(4.4)$ can be rewritten as

$$
\begin{aligned}
0 & =\frac{1}{2} \sigma_{2}^{2} Y_{c c}(c)+\left(\frac{\gamma \rho \sigma_{2}(\alpha-h(\lambda)-\lambda c+\mu)}{\sigma_{1}(1-\gamma)}+g(c)\right) Y_{c}(c)+\frac{\gamma \rho^{2} \sigma_{2}^{2} Y_{c}^{2}(c)}{2(1-\gamma) Y(c)} \\
& +Y(c)\left(\gamma\left[\frac{(\alpha-h(\lambda)-\lambda c+\mu)^{2}}{2(1-\gamma) \sigma_{1}^{2}}-(\alpha-h(\lambda)-\lambda c)\right]-r\right)+(1-\gamma) Y(c)^{\frac{\gamma}{\gamma-1}} .
\end{aligned}
$$

To further simplify (4.7), we set

$$
W(c)=\ln Y(c) .
$$

Moreover, let

$$
\begin{aligned}
& F(c)=\frac{\gamma \rho \sigma_{2}(\alpha-h(\lambda)-\lambda c+\mu)}{\sigma_{1}(1-\gamma)}+g(c), \\
& G(c)=\frac{(\alpha-h(\lambda)-\lambda c+\mu)^{2}}{2(1-\gamma) \sigma_{1}^{2}}-(\alpha-h(\lambda)-\lambda c) \\
& H(c)=\frac{\gamma \rho^{2} \sigma_{2}^{2}}{2(1-\gamma)} .
\end{aligned}
$$

Then, (4.7) can be rewritten as

$$
0=\frac{1}{2} \sigma_{2}^{2} W_{c c}(c)+F(c) W_{c}(c)+\left(H(c)+\frac{\sigma_{2}^{2}}{2}\right) W_{c}^{2}(c)+I(c),
$$

where

$$
I(c)=\gamma G(c)-r+(1-\gamma) e^{\frac{W(c)}{\gamma-1}}
$$

Let $\widetilde{W}(c)$ be a classical solution of (4.8). Then we proceed to verify that the proposed value function

$$
\widetilde{V}(x, c)=\frac{x^{\gamma}}{\gamma} \widetilde{Y}(c),
$$

where

$$
\widetilde{Y}(c)=e^{\widetilde{W}(c)},
$$

equals the value function $V(x, c)$ defined in $(2.15)$.

To obtain the classical solution of (4.8), we will use the subsolution and supersolution method introduced in Section 3.1. Similar to the steps in 3.1, we will first find an ordered pair of subsolution and supersolution $\left(W_{1}(c), W_{2}(c)\right)$. Then we can prove the existence of a classical solution $\widetilde{W}(c)$ of (4.8).

Lemma 4.1. Assume that

$$
r>\gamma \mu-\frac{\gamma(1-\gamma)}{2} \sigma_{1}^{2}
$$

Denote $\tilde{w}$ such that

$$
\tilde{w}=(\gamma-1) \ln \left\{\frac{2}{1-\gamma}\left(r-\gamma\left(\mu-\frac{1-\gamma}{2} \sigma_{1}^{2}\right)\right)\right\} .
$$

Then $\tilde{w}$ is a subsolution of (4.8). 
Proof. Since $\tilde{w}$ is constant,

$$
\frac{1}{2} \sigma_{2}^{2} \tilde{w}_{c c}+F(c) \tilde{w}_{c}+\left(H(c)+\frac{\sigma_{2}^{2}}{2}\right) \tilde{w}_{c}^{2}(c)=0 .
$$

To verify that $\tilde{w}$ is a subsolution of $(4.8)$, it is sufficient to verify $I(c)>0, \forall c \in \mathbb{R}$. Note that

$$
\begin{aligned}
G(c) & =\frac{(\alpha-h(\lambda)-\lambda c+\mu)^{2}}{2(1-\gamma) \sigma_{1}^{2}}-(\alpha-h(\lambda)-\lambda c) \\
& =\frac{\left(\alpha-h(\lambda)-\lambda c+\mu-(1-\gamma) \sigma_{1}^{2}\right)^{2}-\left(\mu-(1-\gamma) \sigma_{1}^{2}\right)^{2}+\mu^{2}}{2(1-\gamma) \sigma_{1}^{2}} \\
& \geq \mu-\frac{(1-\gamma) \sigma_{1}^{2}}{2} .
\end{aligned}
$$

In view of $(4.8)$, we have

$$
\begin{aligned}
I(c) & \geq \gamma\left(\mu-\frac{(1-\gamma) \sigma_{1}^{2}}{2}\right)-r+(1-\gamma) e^{\frac{\tilde{w}}{\gamma-1}} \\
& =\gamma\left(\mu-\frac{(1-\gamma) \sigma_{1}^{2}}{2}\right)-r+2\left(r-\gamma\left(\mu-\frac{1-\gamma}{2} \sigma_{1}^{2}\right)\right) \\
& =r-\gamma\left(\mu-\frac{1-\gamma}{2} \sigma_{1}^{2}\right) \\
& \geq 0 .
\end{aligned}
$$

Then,

$$
\frac{1}{2} \sigma_{2}^{2} \tilde{w}_{c c}+F(c) \tilde{w}_{c}+\left(H(c)+\frac{\sigma_{2}^{2}}{2}\right) \tilde{w}_{c}^{2}(c)+I(c) \geq 0 .
$$

Hence, $\tilde{w}$ is a subsolution of (4.8).

To proceed, we will construct a supersolution of (4.8). Due to the complexity of power utility function, the steps to obtain the supersolution involve more computation. We will split the procedures to several steps.

Lemma 4.2. Let

$$
\begin{aligned}
& n_{1}=2 \sigma_{2}^{2}\left(\frac{\gamma \rho^{2}}{1-\gamma}+1\right), \\
& n_{2}=2 \tilde{\kappa}_{2}-\frac{2 \lambda \gamma \rho \sigma_{2}}{\sigma_{1}(1-\gamma)}, \\
& n_{3}=\frac{\gamma \lambda^{2}}{2(1-\gamma) \sigma_{1}^{2}} .
\end{aligned}
$$

We further assume that $g_{c}(c)<\tilde{\kappa}_{2}$ such that

$$
\tilde{\kappa}_{2}<\frac{2 \lambda \gamma \rho \sigma_{2}}{\sigma_{1}(1-\gamma)}-\frac{\lambda \sigma_{2} \sqrt{\gamma \rho^{2}+1-\gamma}}{\sigma_{1}(1-\gamma)} .
$$

Then the quadratic equation $m^{2} n_{1}+m n_{2}+n_{3}=0$ has two positive real roots denoted by $m_{1}$ and $m_{2}$.

Proof. It is not hard to verify that $n_{1}, n_{3}>0$ and $n_{2}<0$. Moreover,

$$
\begin{aligned}
n_{2}^{2}-4 n_{1} n_{3} & =4\left(\kappa_{2}-\frac{\lambda \gamma \rho \sigma_{2}}{\sigma_{1}(1-\gamma)}\right)^{2}-\frac{4 \lambda^{2} \sigma_{2}^{2}\left(\gamma \rho^{2}+1-\gamma\right)}{\sigma_{1}^{2}(1-\gamma)^{2}} \\
& >0
\end{aligned}
$$


Hence, the quadratic equation $m^{2} n_{1}+m n_{2}+n_{3}=0$ has two positive real roots. We denote the two roots by $m_{1}$ and $m_{2}$.

Lemma 4.3. Let $m_{0}=\left(m_{1}+m_{2}\right) / 2$, then $m_{0}>0$. Assume that

$$
r>K_{1}\left(m_{0}\right),
$$

where $K_{1}\left(m_{0}\right)$ is given by (4.20). Then, define $\widehat{w}(c)$ by

$$
\widehat{w}(c)=m_{0} c^{2}+K_{0},
$$

where $K_{0}$ is a sufficient large constant to guarantee $\widehat{w}(c)>\tilde{w}$, and

$$
K_{0}>(\gamma-1) \ln \left(\frac{r-K_{1}\left(m_{0}\right)}{1-\gamma}\right) .
$$

Then, $\widehat{w}(c)$ is a superslution of (4.8).

Proof. For the $\widehat{w}(c)$ given by (4.15), we have $\widehat{w}_{c}=2 m_{0} c$, and $\widehat{w}_{c c}=2 m_{0}$. Substituting them into (4.8), we have

$$
\begin{aligned}
& \frac{1}{2} \sigma_{2}^{2} \widehat{w}_{c c}+F(c) \widehat{w}_{c}+\left(H(c)+\frac{\sigma_{2}^{2}}{2}\right) \widehat{w}_{c}^{2}(c) \\
& =2 c^{2} m_{0}^{2} \sigma_{2}^{2}\left(\frac{\gamma \rho^{2}}{1-\gamma}+1\right)+m_{0} \sigma_{2}^{2}+2 c m_{0}\left(\frac{\gamma \rho \sigma_{2}(\alpha-h(\lambda)-\lambda c+\mu)}{\sigma_{1}(1-\gamma)}\right)+2 m_{0} g(c) c .
\end{aligned}
$$

On the other hand, in view of (4.13), $\exists \xi \in[0, c]$ such that

$$
g(c)=g(0)+c g_{c}(\xi) \leq g(0)+c \tilde{\kappa}_{2} .
$$

Hence, combining the equations (4.16) and (4.17), we have

$$
\begin{aligned}
\frac{1}{2} & \sigma_{2}^{2} \widehat{w}_{c c}+F(c) \widehat{w}_{c}+\left(H(c)+\frac{\sigma_{2}^{2}}{2}\right) \widehat{w}_{c}^{2}(c) \\
\leq & 2 c^{2} m_{0}^{2} \sigma_{2}^{2}\left(\frac{\gamma \rho^{2}}{1-\gamma}+1\right)+m_{0} \sigma_{2}^{2}+2 c m_{0}\left(\frac{\gamma \rho \sigma_{2}(\alpha-h(\lambda)-\lambda c+\mu)}{\sigma_{1}(1-\gamma)}\right) \\
& +2 m_{0} c\left(g(0)+c \tilde{\kappa}_{2}\right) \\
= & c^{2}\left(m_{0}^{2} n_{1}+m_{0} n_{2}\right)+c m_{0}\left(\frac{2 \gamma \rho \sigma_{2}(\alpha-h(\lambda)+\mu)}{\sigma_{1}(1-\gamma)}+2 g(0)\right)+m_{0} \sigma_{2}^{2} .
\end{aligned}
$$

Furthermore, we have

$$
\begin{aligned}
& \frac{1}{2} \sigma_{2}^{2} \widehat{w}_{c c}+F(c) \widehat{w}_{c}+\left(H(c)+\frac{\sigma_{2}^{2}}{2}\right) \widehat{w}_{c}^{2}(c)+I(c) \\
& \leq c^{2}\left(m_{0}^{2} n_{1}+m_{0} n_{2}\right)+c m_{0}\left(\frac{2 \gamma \rho \sigma_{2}(\alpha-h(\lambda)+\mu)}{\sigma_{1}(1-\gamma)}+2 g(0)\right)+m_{0} \sigma_{2}^{2} \\
& \quad+\frac{\gamma}{2(1-\gamma) \sigma_{1}^{2}}\left[\lambda^{2} c^{2}+\left(\alpha-h(\lambda)+\mu-(1-\gamma) \sigma_{1}^{2}\right)^{2}-2 \lambda c(\alpha-h(\lambda)+\mu\right. \\
& \left.\left.\quad-(1-\gamma) \sigma_{1}^{2}\right)\right]+\gamma \mu-\frac{\gamma(1-\gamma) \sigma_{1}^{2}}{2}-r+(1-\gamma) e^{\frac{\widehat{w}}{\gamma-1}} \\
& =c^{2} \eta_{1}+c \eta_{2}+\eta_{3}-r+(1-\gamma) e^{\frac{\widehat{w}}{\gamma-1}}
\end{aligned}
$$


where

$$
\begin{aligned}
& \eta_{1}=m_{0}^{2} n_{1}+m_{0} n_{2}+n_{3} \\
& \eta_{2}=2 m_{0}\left(\frac{\gamma \rho \sigma_{2}(\alpha-h(\lambda)+\mu)}{\sigma_{1}(1-\gamma)}+g(0)\right)-\frac{\lambda \gamma\left(\alpha-h(\lambda)+\mu-(1-\gamma) \sigma_{1}^{2}\right)}{(1-\gamma) \sigma_{1}^{2}} \\
& \eta_{3}=m_{0} \sigma_{2}^{2}+\frac{\lambda \gamma\left(\alpha-h(\lambda)+\mu-(1-\gamma) \sigma_{1}^{2}\right)^{2}}{2(1-\gamma) \sigma_{1}^{2}}+\gamma \mu-\frac{\gamma(1-\gamma) \sigma_{1}^{2}}{2}
\end{aligned}
$$

Define

$$
K_{1}\left(m_{0}\right)=\eta_{3}-\frac{\eta_{2}^{2}}{4 \eta_{1}}
$$

By virtue of Lemma 4.2 , it is not hard to show that $\eta_{1}<0$. Then we have

$$
\begin{aligned}
& \frac{1}{2} \sigma_{2}^{2} \widehat{w}_{c c}+F(c) \widehat{w}_{c}+\left(H(c)+\frac{\sigma_{2}^{2}}{2}\right) \widehat{w}_{c}^{2}(c)+I(c) \\
& \quad \leq K_{1}\left(m_{0}\right)-r+\left(r-K_{1}\left(m_{0}\right)\right) e^{\frac{m_{0} c^{2}}{\gamma-1}} \\
& \quad=\left(r-K_{1}\left(m_{0}\right)\right)\left(e^{\frac{m_{0} c^{2}}{\gamma-1}}-1\right) \\
& \quad<0 .
\end{aligned}
$$

Furthermore, since $m_{0}<0$, it is easy to verify that

$$
\widehat{w}(c)-\tilde{w}=-2 m_{0} c^{2}+(\gamma-1) \ln \left(\frac{r-K_{1}\left(m_{0}\right)}{1-\gamma}\right)+\left|(\gamma-1) \ln \left(\frac{r-K_{1}\left(m_{0}\right)}{1-\gamma}\right)\right|>0 .
$$

Hence $\widehat{w}(c)$ is a superslution of $(4.8)$ with $\widehat{w}(c)>\tilde{w}$.

Theorem 4.4. There exists a classical solution of equation (4.8) denoted by $\widetilde{W}(c)$ such that

$$
\tilde{w} \leq \widetilde{W}(c) \leq \widehat{w}(c),
$$

where $\tilde{w}$ and $\widehat{w}(c)$ are defined in (4.12) and (4.15), respectively.

The proof of Theorem 4.4 is the same as the proof of Theorem 3.4, so we omit it here.

\subsection{Verification Theorem}

In this section, we focus on the verification theorem. To begin with, we provide a lemma that will be used in verification theorem.

Lemma 4.5. Let

$$
\begin{aligned}
\nu_{1} & =\frac{\lambda^{2}}{2(1-\gamma) \sigma_{1}^{2}}, \\
\nu_{2} & =\lambda \gamma-\frac{\lambda(\alpha-h(\lambda)+\mu) \gamma}{(1-\gamma) \sigma_{1}^{2}} \\
\nu_{3} & =\frac{(\alpha-h(\lambda)+\mu)^{2}}{2(1-\gamma) \sigma_{1}^{2}}-\alpha+h(\lambda) .
\end{aligned}
$$

Assume

$$
\nu_{1}<\frac{\tilde{\kappa}_{2}^{2}}{2 \sigma_{2}^{2}}
$$


and

$$
r>\max \left\{K_{2}\left(\bar{m}_{0}\right), K_{3}\left(\bar{m}_{0}\right)\right\}
$$

where

$$
\begin{aligned}
& \bar{m}_{0}=-\frac{\tilde{\kappa}_{2}}{2 \sigma_{2}^{2}}, \\
& K_{2}\left(\bar{m}_{0}\right)=\delta_{3}\left(\bar{m}_{0}\right)-\frac{\delta_{2}^{2}\left(\bar{m}_{0}\right)}{4 \delta_{1}\left(\bar{m}_{0}\right)}, \\
& K_{3}\left(\bar{m}_{0}\right)=\tilde{\delta}_{3}\left(\bar{m}_{0}\right)-\frac{\tilde{\delta}_{2}^{2}\left(\bar{m}_{0}\right)}{4 \tilde{\delta}_{1}\left(\bar{m}_{0}\right)}, \\
& \delta_{1}\left(\bar{m}_{0}\right)=2 \bar{m}_{0} \sigma_{2}^{2}+2 \tilde{\kappa}_{2} \bar{m}_{0}+2 \nu_{1}, \\
& \delta_{2}\left(\bar{m}_{0}\right)=2 \bar{m}_{0} g(0)+2 \nu_{2}, \\
& \delta_{3}\left(\bar{m}_{0}\right)=\bar{m}_{0} \sigma_{2}^{2}+2 \nu_{3}, \\
& \tilde{\delta}_{1}\left(\bar{m}_{0}\right)=2 \bar{m}_{0} \sigma_{2}^{2}+2 \tilde{\kappa}_{2} \bar{m}_{0}, \\
& \tilde{\delta}_{2}\left(\bar{m}_{0}\right)=2 \bar{m}_{0} g(0), \\
& \tilde{\delta}_{3}\left(\bar{m}_{0}\right)=\bar{m}_{0} \sigma_{2}^{2}+2 m_{0} c^{2}(T),
\end{aligned}
$$

Then we have

$$
E\left[e^{-2 r T+\int_{0}^{T} 4 G(c(s)) d s}\right]<\Theta_{1}
$$

and

$$
E\left[e^{-2 r T+4 \bar{m}_{0} c^{2}(T)}\right]<\Theta_{2},
$$

where $\Theta_{1}$ and $\Theta_{2}$ are constants.

Proof. This is a direct corollary of Lemma 4.7 of Fleming and Pang (2004). We omit the details here.

Now we will establish the verification theorem for the optimal debt ratio and dividend payment policies.

Theorem 4.6. Suppose there exists a function $\widetilde{W}(t)$ defined in Theorem 4.4 such that $(4.9)$ and (4.22) hold, and that $\widetilde{Y}(t)$ solves (4.8). Assume that (4.11), (4.14), (4.23), (4.24) hold. Let

$$
\widetilde{V}(x, c)=\frac{1}{r} \ln x+\tilde{Y}(c) .
$$

Then,

(a) For all admissible pairs of control policies $u=(\pi, z) \in \mathcal{A}$,

$$
\widetilde{V}(x, c) \geq J(x, c, u)=E_{x, c} \int_{0}^{\infty} e^{-r t} \frac{1}{\gamma}(z(t) X(t))^{\gamma} d t .
$$

(b) If $u^{*}=\left(\pi^{*}, z^{*}\right)$ satisfies the following:

$$
\begin{aligned}
& \pi^{*}=\frac{\alpha-h(\lambda)-\lambda c+\mu+(\gamma-1) \sigma_{1}^{2}}{(1-\gamma) \sigma_{1}^{2}}+\frac{\tilde{Y}_{c}(c) \rho \sigma_{2}}{(1-\gamma) \tilde{Y}(c) \sigma_{1}}, \\
& z^{*}=\tilde{Y}(c)^{\frac{1}{\gamma-1}} .
\end{aligned}
$$


Then $u^{*} \in \mathcal{A}$. We will have

$$
\widetilde{V}(x, c) \geq J\left(x, c, u^{*}\right)=E_{x, c} \int_{0}^{\infty} e^{-r t} \frac{1}{\gamma}(z(t) X(t))^{\gamma} d t .
$$

Moreover, $\widetilde{V}(x, c)$ is the value function defined in $(2.15)$. That is, $\widetilde{V}(x, c)=V(x, c)$.

Proof. Similar to Theorem 4.6, applying Itô's lemma to $e^{-r t} \widetilde{V}(x, c)$, we have

$$
\begin{aligned}
d\left(e^{-r t} \widetilde{V}(X(t), c(t))\right) & =e^{-r t} d \widetilde{V}(X(t), c(t))-r e^{-r t} \widetilde{V}(X(t), c(t)) d t \\
& =e^{-r t}\left[\left(\mathcal{L}^{u} \widetilde{V}(X(t), c(t))-r \widetilde{V}(X(t), c(t))\right) d t+d\left(\bar{M}_{1}+\bar{M}_{2}\right)\right]
\end{aligned}
$$

where $\bar{M}_{1}$ and $\bar{M}_{2}$ are defined in (3.27). In view of (2.18), for any control $u \in \mathcal{A}$, we have

$$
\mathcal{L}^{u} \widetilde{V}(X(t), c(t))-r \widetilde{V}(X(t), c(t)) \leq-\frac{1}{\gamma}(z(t) X(t))^{\gamma} .
$$

Integrating (4.31) in $[0, T]$ and taking expectation on both sides, we have

$$
E \int_{0}^{T} d\left(e^{-r t} \widetilde{V}(X(t), c(t))\right) \leq-E \int_{0}^{T} e^{-r t} \frac{1}{\gamma}(z(t) X(t))^{\gamma} d t .
$$

Then,

$$
E e^{-r T} \widetilde{V}(X(T), c(T))-\widetilde{V}(x, c) \leq-E \int_{0}^{T} e^{-r t} \frac{1}{\gamma}(z(t) X(t))^{\gamma} d t
$$

Hence,

$$
\begin{aligned}
\widetilde{V}(x, c) & \geq E \int_{0}^{T} e^{-r t} \frac{1}{\gamma}(z(t) X(t))^{\gamma} d t+E e^{-r T} \widetilde{V}(X(T), c(T)) \\
& =E \int_{0}^{T} e^{-r t} \frac{1}{\gamma}(z(t) X(t))^{\gamma} d t+E e^{-r T}\left[\frac{x^{\gamma}}{\gamma} \widetilde{Y}(c(T))\right] \\
& =E \int_{0}^{T} e^{-r t} \frac{1}{\gamma}(z(t) X(t))^{\gamma} d t+E e^{-r T}\left[\frac{x^{\gamma}}{\gamma} e^{\widetilde{W}(c(T))}\right] .
\end{aligned}
$$

It is not hard to show that

$$
\limsup _{T \rightarrow \infty} E e^{-r T} \tilde{V}(X(T), c(T)) \geq 0
$$

Then (4.30) is verified.

Consider the debt ratio and dividend payment rate strategies $u^{*}=\left(\pi^{*}, z^{*}\right)$,

$$
\begin{aligned}
& \pi^{*}=\frac{\alpha-h(\lambda)-\lambda c+\mu+(\gamma-1) \sigma_{1}^{2}}{(\gamma-1) \sigma_{1}^{2}}+\frac{\tilde{Y}_{c}(c) \rho \sigma_{2}}{(\gamma-1) \widetilde{Y}(c) \sigma_{1}}, \\
& z^{*}=\tilde{Y}(c)^{\frac{1}{\gamma-1}} .
\end{aligned}
$$

It is not hard to show that

$$
\begin{aligned}
& \pi^{*} \in \arg \max _{\pi}\left\{\frac{1}{2}(\gamma-1) Y(c) \sigma_{1}^{2} \pi^{2}+\pi\left[Y(c)\left(\alpha-h(\lambda)-\lambda c+\mu+(\gamma-1) \sigma_{1}^{2}\right)+Y_{c}(c) \rho \sigma_{1} \sigma_{2}\right]\right\}, \\
& z^{*} \in \arg \max _{z}\left[-z Y(c)+\frac{1}{\gamma} z^{\gamma}\right] .
\end{aligned}
$$


Then $u^{*} \in \mathcal{A}$. Let $\left(X^{*}(t), c^{*}(t)\right)$ be the corresponding trajectories of $u^{*}$. We have

$$
\mathcal{L}^{u^{*}} \widetilde{V}\left(X^{*}(t), c^{*}(t)\right)-r \widetilde{V}\left(X^{*}(t), c^{*}(t)\right)=-\frac{1}{\gamma}\left(z^{*}(t) X^{*}(t)\right)^{\gamma} .
$$

Hence,

$$
\widetilde{V}(x, c)=E \int_{0}^{T} e^{-r t} \frac{1}{\gamma}\left(z^{*}(t) X^{*}(t)\right)^{\gamma} d t+E e^{-r T} \widetilde{V}\left(X^{*}(T), c^{*}(T)\right) .
$$

To prove (4.29), we need to verify

$$
\widetilde{V}(x, c) \leq E \int_{0}^{\infty} e^{-r t} \frac{1}{\gamma}\left(z^{*}(t) X^{*}(t)\right)^{\gamma} d t .
$$

That is, it is sufficient to show that

$$
\liminf _{T \rightarrow \infty} E e^{-r T} \widetilde{V}\left(X^{*}(T), c^{*}(T)\right) \leq 0 .
$$

For simplicity, let

$$
\psi=\pi^{*}(t)(\alpha-h(\lambda)-\lambda c(t)+\mu(t))+\mu(t)-\frac{1-2 \gamma}{2}\left(\pi^{*}(t)+1\right)^{2} \sigma_{1}^{2} .
$$

Therefore, (2.11) can be rewritten as

$$
d X^{*}(t)=\left(\psi+\frac{1-2 \gamma}{2}\left(\pi^{*}(t)+1\right)^{2} \sigma_{1}^{2}-z^{*}(t)\right) X(t) d t+\left(\pi^{*}(t)+1\right) \sigma_{1} X(t) d W_{1}(t) .
$$

Then, we have

$$
X^{*}(T)=x \exp \left(\int_{0}^{T}\left(\psi-z^{*}(t)-\gamma\left(\pi^{*}(t)+1\right)^{2} \sigma_{1}^{2}\right) d t+\int_{0}^{T}\left(\pi^{*}(t)+1\right) \sigma_{1} d W_{1}(t)\right) .
$$

Hence, the Cauchy-Schwarz inequality yields

$$
\begin{aligned}
E & e^{-r T} \widetilde{V}\left(X^{*}(T), c^{*}(T)\right) \\
& =E e^{-r T} \frac{X^{*}(T)^{\gamma}}{\gamma} \widetilde{Y}\left(c^{*}(T)\right) \\
& =\frac{x^{\gamma}}{\gamma} E\left\{e^{\left[\int_{0}^{T}\left(\gamma\left(\psi-z^{*}(t)-\gamma\left(\pi^{*}(t)+1\right)^{2} \sigma_{1}^{2}\right)-r\right) d t+\int_{0}^{T} \gamma\left(\pi^{*}(t)+1\right) \sigma_{1} d W_{1}(t)\right]} \tilde{Y}\left(c^{*}(T)\right)\right\} \\
& \leq \frac{x^{\gamma}}{\gamma}\left(E\left[e^{\int_{0}^{T} 2\left(\gamma\left(\psi-z^{*}(t)\right)-r\right) d t} \widetilde{Y}^{2}\left(c^{*}(T)\right)\right]\right)^{\frac{1}{2}} \times\left(E e^{\int_{0}^{T}\left[2 \gamma\left(\pi^{*}(t)+1\right) \sigma_{1} d W_{1}(t)-2 \gamma^{2}\left(\pi^{*}(t)+1\right)^{2} \sigma_{1}^{2} d t\right]}\right)^{\frac{1}{2}} \\
& \leq \frac{x^{\gamma}}{\gamma}\left(E\left[e^{\int_{0}^{T} 2\left(\gamma\left(\psi-z^{*}(t)\right)-r\right) d t} \widetilde{Y}^{2}\left(c^{*}(T)\right)\right]\right)^{\frac{1}{2}} .
\end{aligned}
$$

On the other hand, we have $z^{*}(t)>0$ and

$$
\begin{aligned}
\psi-G(c)= & \pi^{*}(t)(\alpha-h(\lambda)-\lambda c(t)+\mu(t))+\mu(t)-\frac{1-2 \gamma^{2}}{2}\left(\pi^{*}(t)+1\right)^{2} \sigma_{1}^{2} \\
& -\frac{(\alpha-h(\lambda)-\lambda c+\mu)^{2}}{2(1-\gamma) \sigma_{1}^{2}}+(\alpha-h(\lambda)-\lambda c) \\
= & -\frac{1}{2(1-\gamma) \sigma_{1}^{2}}\left[(1-\gamma)\left(\pi^{*}(t)+1\right) \sigma_{1}-(\alpha-h(\lambda)-\lambda c+\mu)\right]^{2} \\
\leq & 0
\end{aligned}
$$


Therefore, we obtain

$$
\begin{aligned}
& E\left[e^{\int_{0}^{T} 2\left(\gamma\left(\psi-z^{*}(t)\right)-r\right) d t} \tilde{Y}^{2}\left(c^{*}(T)\right)\right] \\
& \leq E\left[e^{\int_{0}^{T} 2(\gamma G(c)-r) d t} \widetilde{Y}^{2}\left(c^{*}(T)\right)\right] \\
& \leq e^{-2 r T} E\left[e^{\int_{0}^{T} 4(\gamma G(c)-r) d t}\right]^{\frac{1}{2}} E\left[\widetilde{Y}^{4}\left(c^{*}(T)\right)\right]^{\frac{1}{2}}
\end{aligned}
$$

Let

$$
\epsilon=\max \left\{\gamma \mu-\frac{\gamma(1-\gamma)}{2} \sigma_{1}^{2}, K_{1}\left(m_{0}\right), K_{2}\left(\bar{m}_{0}\right), K_{3}\left(\bar{m}_{0}\right)\right\} .
$$

Define $\bar{\epsilon}=r-\epsilon$ and $\bar{r}=\frac{1}{2}(r+\epsilon)$. Then $\bar{\epsilon}>0$ and $r>\bar{r}>\epsilon$. Thus, by virtue of Lemma 4.5, we obtain

$$
\begin{aligned}
& e^{-2 r T} E\left[e^{\int_{0}^{T} 4(\gamma G(c)-r) d t}\right]^{\frac{1}{2}} E\left[\widetilde{Y}^{4}\left(c^{*}(T)\right)\right]^{\frac{1}{2}} \\
& \quad=e^{-\bar{\epsilon} T} E\left[e^{-2 \bar{r} T} e^{\left.\int_{0}^{T} 4(\gamma G(c)-r) d t\right]^{\frac{1}{2}} E} E\left[e^{-2 \bar{r} T} e^{4 \widetilde{W}(c)}\right]^{\frac{1}{2}}\right. \\
& \leq e^{-\bar{\epsilon} T} \Theta_{1}^{\frac{1}{2}} E\left[e^{-2 \bar{r} T} e^{4 m_{0} c^{2}(T)+4 K_{0}}\right]^{\frac{1}{2}} \\
& \leq \Lambda e^{-\bar{\epsilon} T} \Theta_{1}^{\frac{1}{2}} \Theta_{2}^{\frac{1}{2}},
\end{aligned}
$$

where $\Lambda$ is a constant. Hence,

$$
\liminf _{T \rightarrow \infty} E e^{-r T} \widetilde{V}\left(X^{*}(T), c^{*}(T)\right) \leq \liminf _{T \rightarrow \infty} \Lambda e^{-\bar{\epsilon} T} \Theta_{1}^{\frac{1}{2}} \Theta_{2}^{\frac{1}{2}}=0 .
$$

Thus, (4.36) is satisfied. Combining with (4.30) and (4.36), we have

$$
V(x, c)=E \int_{0}^{\infty} e^{-r t} \frac{1}{\gamma}\left(z^{*}(t) X^{*}(t)\right)^{\gamma} d t
$$

Then $(b)$ is proved.

\section{An Example}

In this section, we will find the explicit solution for a special stochastic process. Assume the claim rate $c(t)$ follows the mean-reverting process. That is, we assume

$$
\left\{\begin{array}{l}
d c(t)=\theta(\bar{c}-c(t)) d t+\sigma_{2} d W_{2}(t), \\
c(0)=c,
\end{array}\right.
$$

where $\bar{c}$ represents the expectation of claim rate of an insurance economy, and $\theta>0$ represents the speed of reversion to $\bar{c}$. Assume that $\bar{c}>0 . \sigma_{2} d W_{2}(t)$ represents the random shocks of claims due to the financial market and other economic performance.

\subsection{Value Function and Optimal Strategies}

We will present the explicit form of the value function in logarithm utility function in this section. By using the dynamic programming principle, (2.19) can be written as

$$
\begin{aligned}
& \max _{\pi}\left[\frac{1}{2} V_{x x} \sigma_{1}^{2}(\pi+1)^{2} x^{2}+V_{x c} \rho \sigma_{1} \sigma_{2} x(\pi+1)+V_{x} \pi(\alpha-h(\lambda)-\lambda c+\mu)\right] \\
& \quad+\max _{z}\left[-z x V_{x}+\ln (z x)\right]+\frac{1}{2} \sigma_{2}^{2} V_{c c}+\mu x V_{x}+\theta(\bar{c}-c(t)) V_{c}-r V(x, c)=0 .
\end{aligned}
$$


The optimal debt ratio and dividend payment policies $u^{*}=\left(\pi^{*}, z^{*}\right)$ follows

$$
\begin{aligned}
& \pi^{*}=\frac{\alpha-h(\lambda)-\lambda c+\mu-\sigma_{1}^{2}}{\sigma_{1}^{2}}, \\
& z^{*}=r .
\end{aligned}
$$

By virtue of (3.4), we can construct the value function $V(x, c)$ as

$$
V(x, c)=\frac{1}{r} \ln x+Y(c) .
$$

$Y(c)$ is the solution of the following equation

$$
\frac{1}{2} \sigma_{2}^{2} Y_{c c}(c)+\theta(\bar{c}-c(t)) Y_{c}(c)-r Y(c)+N(c)=0,
$$

where $N(c)$ is defined in (3.7). Consider a solution with the form

$$
Y(c)=B_{1} c^{2}+B_{2} c+B_{3} .
$$

Substituting (5.6) to (5.5), we have

$$
\begin{aligned}
0= & c^{2}\left[-B_{1}(2 \theta+r)+\frac{\lambda^{2}}{2 r \sigma_{1}^{2}}\right]+c\left[-B_{2}(\theta+r)+2 B_{1} \theta \bar{c}-\frac{\lambda(\alpha-h(\lambda)+\mu)}{r \sigma_{1}^{2}}+\frac{\lambda}{r}\right] \\
& +\theta \bar{c} B_{2}-r B_{3}+\frac{(\alpha-h(\lambda)+\mu)^{2}}{2 r \sigma_{1}^{2}}+\frac{\sigma_{1}^{2}-2(\alpha-h(\lambda))}{2 r}+\ln r-1-\frac{1}{2 r} \sigma_{1}^{2}+\frac{\lambda^{2} \sigma_{2}^{2}}{2 r \sigma_{1}^{2}(2 \theta+r)} .
\end{aligned}
$$

Then, the coefficients of $Y(c)$ can be verified. That is,

$$
\begin{aligned}
B_{1}= & \frac{\lambda^{2}}{2 r \sigma_{1}^{2}(2 \theta+r)}, \\
B_{2}= & \frac{1}{r \sigma_{1}^{2}(\theta+r)}\left(\frac{\lambda^{2} \theta \bar{c}}{2 \theta+r}-\lambda\left(\alpha-h(\lambda)+\mu-\sigma_{1}^{2}\right)\right) \\
B_{3}= & \frac{1}{r}\left(\frac{\lambda^{2} \theta \bar{c}\left(\theta \bar{c}-\lambda\left(\alpha-h(\lambda)+\mu-\sigma_{1}^{2}\right)(2 \theta+r)\right)}{r \sigma_{1}^{2}(\theta+r)(2 \theta+r)}+\frac{(\alpha-h(\lambda)+\mu)^{2}}{2 r \sigma_{1}^{2}}\right. \\
& \left.+\frac{\sigma_{1}^{2}-2(\alpha-h(\lambda))}{2 r}+\ln r-1-\frac{1}{2 r} \sigma_{1}^{2}+\frac{\lambda^{2} \sigma_{2}^{2}}{2 r \sigma_{1}^{2}(2 \theta+r)}\right) .
\end{aligned}
$$

Thus, we have

$$
V(x, c)=\frac{1}{r} \ln x+B_{1} c^{2}+B_{2} c+B_{3} .
$$

The verification theorem guaranteed that $V(x, c)$ is the value function and the corresponding optimal policies is $u^{*}$.

\subsection{Ruin Probability}

We will study the impact of the optimal debt ratio and dividend payment strategies on the ruin probability. Let $\pi^{*}$ and $z^{*}$ be the optimal debt ratio and dividend payment strategies obtained in (5.3), respectively. Let $q(t)=\alpha-h(\lambda)-\lambda c(t)+\mu(t)$. By choosing the optimal strategies, (2.11) can be rewritten as 


$$
\begin{aligned}
\frac{d X(t)}{X(t)} & =\left[\pi^{*}(\alpha-h(\lambda)-\lambda c(t)+\mu(t))+\mu(t)-z^{*}(t)\right] d t+\left(\pi^{*}+1\right) \sigma_{1} d W_{1}(t), \\
& =\left[\frac{q^{2}(t)}{\sigma_{1}^{2}}-q(t)+\mu(t)-r\right] d t+\frac{q(t)}{\sigma_{1}} d W_{1}(t) .
\end{aligned}
$$

Hence,

$$
X(t)=x \exp \left\{\int_{0}^{t}\left[\frac{q^{2}(s)}{2 \sigma_{1}^{2}}-q(s)+\mu(s)-r\right] d s+\int_{0}^{t} \frac{q(s)}{\sigma_{1}} d W_{1}(s)\right\} .
$$

Note that $X(t)$ follows a geometric Brownian motion process when optimal controls are adopted. Then $X(t)>0$ and $\mathbb{P}(\tau<\infty)=0$ since $x>0$. It shows that financial ruin can be completely avoided if optimal debt management is executed. This is because the optimal debt ratio sets a constraint on the written liability size such that the written liability $L(t)$ should depend on the surplus status $X(t)$. In the Cramér-Lundberg model with dividends payments, when total discounted dividend payment is maximized, the company will almost surely be financial ruined. In our model, when liability size is well managed with optimal debt ratio, the financial ruin can be immunized from the claim rate shocks, even though the total discounted dividend payment is maximized. This is one of the advantages of our model. Intuitively, when surplus is low, the company should chooses to write much less new policies to monitor the risk of financial ruin.

\subsection{Impact of Reinsurance}

In this section, we analyze the impact on the optimal debt ratio of the insurance companies in two types of utility functions due to reinsurance. Denote by $\pi^{l}$ and $\pi^{p}$ the optimal debt ratios in the cases of logarithm utility and power utility. In view of (3.24) and (4.29), we have

$$
\begin{aligned}
\pi^{l} & =\frac{\alpha-h(\lambda)-\lambda c+\mu-\sigma_{1}^{2}}{\sigma_{1}^{2}} \\
\pi^{p} & =\frac{\alpha-h(\lambda)-\lambda c+\mu+(\gamma-1) \sigma_{1}^{2}}{(1-\gamma) \sigma_{1}^{2}}+\frac{\widetilde{Y}_{c}(c) \rho \sigma_{2}}{(1-\gamma) \widetilde{Y}(c) \sigma_{1}}
\end{aligned}
$$

$\widetilde{Y}(c)$ is independent of $\lambda$. In addition, since $\bar{c}$ represents the expectation of claim rate of a wellperformed insurance company in the long-term period, $\bar{c}$ is naturally assumed to be greater than 0 . The retention level $\lambda$ is assumed to be positive. The reinsurance cost $h(\lambda)$ is negatively correlated to the retention level. Thus $h_{\lambda}(\lambda)<0$. By simple calculation, it is not hard to find that

$$
\begin{aligned}
\pi_{\lambda}^{l} & =\frac{-h_{\lambda}-c}{\sigma_{1}^{2}}, \\
\pi_{\lambda}^{p} & =\frac{-h_{\lambda}-c}{(1-\gamma) \sigma_{1}^{2}} .
\end{aligned}
$$

In our model we assume the claim rate follows a mean reverting process in the long run for tractability. The claim rate reverts to a positive mean $\bar{c}$ with small random shocks.

$$
\begin{aligned}
& E\left[\pi_{\lambda}^{l}\right]=\frac{-h_{\lambda}-\bar{c}}{\sigma_{1}^{2}}, \\
& E\left[\pi_{\lambda}^{p}\right]=\frac{-h_{\lambda}-\bar{c}}{(1-\gamma) \sigma_{1}^{2}} .
\end{aligned}
$$


Hence, we can observe that the optimal debt ratio relies on the sensitivity of reinsurance cost. When $-h_{\lambda}$ is sufficient high, that is, a small increase of retention level will significantly decrease the reinsurance cost, the insurance company is suggested to write more liability contracts if retention level is higher. Otherwise, if increasing the retention level can't significantly reduce the reinsurance cost, the insurance company will choose to lower the debt level to maximize the utilities of dividend payments. From the above analysis, we have shown that the reinsurance policy has obvious impact on insurance companies' debt management.

\section{Concluding Remarks}

In this paper, we derived the optimal debt ratio and dividend optimization of an insurance company taking into account the reinsurance policies. The claim rate is assumed to be risky and unpredictable. Incorporating the impact of reinsurance on the financial status of the insurance companies, we aim to maximize the total expected discounted utility of dividend in the infinite time horizon in the logarithm and power utility cases, respectively. A generalized diffusion process of surplus is presented. By using the dynamic programming approach, we derive the associated HJB equation. Furthermore, we adopt the subsolution-supersolution method to solve for the stochastic control problem and obtain the explicit classical solution of value function and corresponding optimal debt ratio and dividend strategies under simple condition. The economic insights shown in the example provide guidance for decision makers in government or industries to manage the leverage level and dividend policies.

Note that we are considering an unconstrained optimization problem. The insurance company has no constraint on the debt ratio. Setting thresh-holds for debt ratios of insurance companies is more realistic but make the problem more complicated. The current paper focuses on the hyperbolic absolute risk aversion (HARA) type utility function, for which we can obtain the closedform solution in logarithm utility and the power utility. For our future research, we plan to treat the exponential utility function, which will lead to a different type of solutions with added difficulties in finding value function and proving the verification theorem. In future study, we will also consider the debt ratio and dividend constraints in various situations. Moreover, we can consider the interest rate shocks. The interest rate fluctuates from time to time and is unlikely to be deterministic. In addition, multiple assets in the investment could be studied. Hence, the stochastic control problem will be more versatile. Although the HJB equation will be derived in routine and subsolutionsupersolution can be used to solve for the value function, we need overcome the difficulty that ordered pair of subsolution and supersolution may not have simple explicit expressions, which will add difficulties to find the analytic solutions.

Furthermore, to better reflect the reality, regime-switching models for the stochastic processes such as asset values, claim rate, and interest rate can be considered. The regime-switching models are known able to capture the extreme economic movement such as market changes. For example, the asset values may have different drift and volatilities in different types of markets. The switchings among different markets can be described by a continuous-time Markov chain. Using the usual dynamic programming approach together with the use of properties of regime-switchings, the value function obeys a coupled system of HJB equations. Thus, the model becomes more versatile but more complicated. Solving the coupled system of HJB equations analytically is very difficult. Nevertheless, numerical approximation method can provide a viable alternative. 


\section{Acknowledgments}

We are grateful to the anonymous referee for his/her valuable comments and suggestions. This research was supported in part by Faculty Research Grant by The University of Melbourne. The research of H. Yang was supported in part by Research Grants Council of the Hong Kong Special Administrative Region (project No. HKU 705313P) and Society of Actuaries' Centers of Actuarial Excellence Research Grant. The research of G. Yin was supported in part by the National Science Foundation under DMS-1207667.

\section{References}

Asmussen, S., Høgaard, B., and Taksar, M. (2000). Optimal risk control and dividend distribution policies. Example of excess-of loss reinsurance for an insurance corporation. Finance and Stochastics, 4: 299-324.

Asmussen, S. and Taksar, M. (1997). Controlled diffusion models for optimal dividend pay-Out. Insurance: Mathematics and Economics, 20: 1-15.

Bai, L. H. and Guo, J. Y. (2008). Optimal proportional reinsurance and investment with multiple risky assets and no-shorting constraint. Insurance: Mathematics and Economics, 42: 968-975.

Bai, L. H., Cai, J. and Zhou, M. (2008). Optimal reinsurance policies for an insurer with a bivariate reserve risk process in a dynamic setting, Insurance: Mathematics and Economics, 53: 664-670.

Choulli, T., Taksar, M. and Zhou, X. Y. (2001). Excess-of-loss reinsurance for a company with debt liability and constraints on risk reduction. Quant. Finance 1:573-96.

De Finetti, B. (1957). Su unimpostazione alternativa della teoria collettiva del rischio. Transactions of the XVth International Congress of Actuaries 2: 433-443.

Fleming, W. H. and Pang, T. (2004). An application of stochastic control theory to financial economics, SIAM Journal of Control and Optimization, 43(2): 502-531.

Gerber, H. U. and Shiu, E. S. W. (2004). Optimal dividends: analysis with Brownian motion. North American Actuarial Journal, 8: 1-20.

Gerber, H. U. and Shiu, E. S. W. (2006). On optimal dividend strategies in the compound Poisson model. North American Actuarial Journal, 10: 76-93.

Jin, Z., Yin, G., Wu, F. (2013). Optimal reinsurance strategies in regime-switching jump diffusion models: stochastic differential game formulation and numerical methods, Insurance: Mathematics and Economics, 53: $733-746$.

Jin, Z., Yang, H., Yin, G. (2013). Numerical methods for optimal dividend payment and investment strategies of regime-switching jump diffusion models with capital injections, Automatica, 49(8): 2317-2329.

Kulenko, N. and Schimidli, H. (2008). An optimal dividend strategy in a Cramer Lundberg model with capital injections. Insurance: Mathmatics and Economics, 43: 270-278.

Lundberg, F. (1903). Approximerad Framställning av Sannolikehetsfunktionen, Aterförsäkering av Kollektivrisker, Almqvist \& Wiksell, Uppsala. Akad. Afhandling. Almqvist o. Wiksell, Uppsala.

Meng, H., Siu, T.K. (2011). Optimal mixed impulse-equity insurance control problem with reinsurance. SIAM Journal on Control and Optimization 49(1): 254-279.

Meng, H., Siu, T.K., Yang, H. (2013). Optimal dividends with debts and nonlinear insurance risk processes. Insurance: Mathematics and Economics 53: 110-121.

Øksendal, Bernt (2003). Stochastic Differential Equations: An Introduction with Applications. Springer, Berlin.

Pang, T. (2006). Stochastic portfolio optimization with log utility, International Journal of Theoretical and Applied Finance, 9(6): 869-887. 
Pao, C. V. (1992). Nonlinear Parabolic and Elliptic Equations. Plenum Press, New York.

Stein, Jerome L. (2012). Stochastic Optimal Control and the U.S. Financial Debt Crisis. Springer, New York.

Wei, J., Yang, H., Wang, R. (2010). Classical and impulse control for the optimization of dividend and proportional reinsurance policies with regime switching, Journal of Optimization Theory and Applications, 147: 358-377.

Yao, D., Yang, H. and Wang, R. (2011). Optimal dividend and capital injection problem in the dual model with proportional and fixed transaction costs. European Journal of Operational Research, 211: 568-576.

Zhang, X., Siu, T.K. (2012). On optimal proportional reinsurance and investment in a Markovian regimeswitching economy, Acta Mathematica Sinica, English Series, 27: 1-16. 